\title{
ERRATA
}

\section{Erratum: Semiclassical theory of the Coulomb anomaly [JETP Lett. 66, No. 3, 214-221 (10 August 1997)]}

S. Levitov and A. V. Shytov

Massachusetts Institute of Technology, Cambridge, MA 02139, USA; L. D. Landau

Institute of Theoretical Physics Russian Academy of Sciences, 117334 Moscow, Russia

[S0021-3640(97)01319-4]

PACS numbers: 73.23.Hk, 73.40.Gk, 72.15.Qm, 99.10.+g

The first author's name should be as follows: "L. S. Levitov." 